\title{
BRST Cohomology for Certain Reducible Topological Symmetries
}

\author{
Sophie Chemla, Jaap Kalkman \\ Rijkuniversiteit Utrecht, Mathematisch Instituut, Postbus 80.010, 3508 TA Utrecht, \\ The Netherlands. e. mail: chemla@math.ruu.nl and kalkman@math.run.nl
}

Received: 15 March 1993/in revised form: 19 July 1993

\begin{abstract}
In this paper, two different definitions of the BRST complex are connected. We obtain the BRST complex of topological quantum field theories (leading to equivariant cohomology) from the standard definition of the classical BRST complex (leading to Lie algebra cohomology) provided that we include ghosts for ghosts. Hereby, we use a finite dimensional model with a semi-direct product action of $H \bowtie \operatorname{Diff} M$ on a configuration space $M$, where $H$ is a compact Lie group representing the gauge symmetry in this model.
\end{abstract}

\section{Introduction}

It is well known that the BRST cohomology of a field theory with gauge group $G$ equals the Lie algebra cohomology of Lie $(G)$ with values in the functionals on the space of fields. Several years ago it was shown by Henneaux and others ([H, K-S $]$ ) that this is also true for the BRST cohomology of finite dimensional Hamiltonian systems. In the case where the $G$-action is not free on some open set, the differential algebra of the BRST complex has to be enlarged with so-called ghosts for ghosts to obtain cohomology classes with a physical meaning (see e.g, [F-H-S-T]).

On the other hand it is well known that the BRST cohomology of cohomological field theories ([Wi]) with additional gauge symmetries equals equivariant cohomology, thus giving the cohomology of all kinds of moduli spaces. However, as described in [O-S-vB] and [Ka], the differential leading to this cohomology is not the standard equivariant differential.

This paper grew out from the uneasy feeling that it is not good to have several disconnected definitions of the BRST complex. In the subsequent sections we will link the two different definitions mentioned above. Furthermore, we will explain the non-standard equivariant differential using results of [F-H-S-T]. To obtain this, we start from a $G$-action on a manifold $M$, where $G$ is the semidirect product of a compact Lie group $H$ and $\operatorname{Diff}(M)$ (the group of diffeomorphisms of $M$ ). This theory is topological in the sense that there is only one gauge orbit. 
As in [Ka], we choose a finite dimensional setting, meaning that both $M$ and $H$ are finite dimensional. This makes it mathematically more tractable and has furthermore the advantage that we do not need to bother whether to take local cohomology (as is usual in field theory) or not.

All along this article, we will use the following notations: If $M$ is a manifold, then $T M$ will be its tangent bundle. If $\mathcal{F}$ is a vector bundle over $M$, then $\Gamma(M, \mathcal{F})$ will denote its global sections. Let $A$ be a commutative, associative $\mathbb{R}$-algebra with unity. We will write $\operatorname{Der}(A)$ for the derivations of $A$. If $V$ is a $A$-module, then $S_{A}(V)$ will be the symmetric algebra of $V$ and $S^{n}(V)$ its $n^{\text {th }}$ component.

\section{The Classical BRST Charge}

The aim of this section is to describe BRST theory associated to a symplectic manifold $N$, called the phase space, and a set of first class constraints. First class constraints are just functions on $N$ satisfying certain conditions. The zero set of these functions is called the constraint manifold. In the sequel we will write constraints as a short-hand for first class constraints. BRST theory assigns to a given set of constraints a so-called BRST charge $Q$. This charge $Q$ is an element of a super Poisson algebra extension $\mathcal{B}$ of $C^{\infty}(N) . Q$ is a natural and geometric object in the sense that if the constraint manifold is represented by another set of constraints, then the two BRST charges are related by a super canonical transformation.

Let $(N, \omega)$ be a symplectic manifold. Then $C^{\infty}(N)$ is a Poisson algebra with Poisson bracket $\{\cdot, \cdot\}$. A collection of functions $f_{\alpha}$ is called a set of first class constraints if

$$
\left\{f_{\alpha}, f_{\beta}\right\}=c_{\alpha \beta}^{\gamma} f_{\gamma}
$$

for certain $c_{\alpha \beta}^{\gamma} \in C^{\infty}(N)$. For simplicity we will assume that $N$ is finite dimensional and that $f_{\alpha}$ is a finite collection of functions. For the moment we will moreover assume that the constraints are regular, that is to say the constraint manifold $Z$ is an honest submanifold of $N$ of codimension equal to the number of constraints. Condition (1) implies that the ideal $I \subset C^{\infty}(N)$ generated by the constraints is a Poisson subalgebra.

In the case of a Lie group $H$ acting Hamiltonially on $N$, the components of the associated momentum map satisfy (1). The $c_{\alpha \beta}^{\gamma}$ are constants in this case equal to the structure constants of the Lie algebra of $H$.

Symplectic reduction entails the following. The constraints give rise to vector fields via the Poisson bracket. From the regularity condition it follows that these vector fields, when restricted to $Z$, span a subbundle of the tangent bundle $T Z$ of $Z$ and from condition (1) it follows that this subbundle is involutive. Note that this is only true on $Z$. On $N$ the vector fields are not involutive in general, they need not even span a subbundle. One says that the symmetry algebra closes only on $Z$. Using Frobenius' theorem we obtain a foliation of $Z$. Thus $Z$ is locally a fiber bundle. If this fibration persists globally then the space of fibers $X$ is called the symplectic quotient of $\left(N, \omega, f_{\alpha}\right) . X$ is also called reduced phase space and the fibers are sometimes referred to as gauge orbits by physicists. It is important to know its structure because often the dynamics of a physical system can be described on this lower dimensional reduced phase space.

In the case of a Lie group $H$ acting freely and Hamiltonially on $N$, this symplectic reduction is called Marsden-Weinstein reduction. If $\mu$ denotes the momentum 
map then the symplectic quotient $X$ equals $\mu^{-1}(0) / H$. BRST theory, however, is much more general. It can handle constraints not coming from any group action, or constraints coming from non-free group actions. It is this last case that we shall deal with in the next sections.

The idea of (classical) BRST theory is to obtain the ring of functions on the reduced phase space $X$ by extending the Poisson Algebra $C^{\infty}(N)$ to a super Poisson algebra $\mathcal{B}$ and then compute the cohomology of an appropriate differential, the BRST operator $D$.

Let $m$ be the number of constraints $f_{\alpha}$. They form a map $F: N \rightarrow V^{*}$, where $V^{*}=\mathbf{R}^{m}$. By definition $Z=\{x \in N \mid F(x)=0\}$. The condition that the constraints are regular (or independent) is equivalent with the condition that 0 is a regular value of the map $F$. The BRST algebra (see e.g. $[\mathrm{H}]$ ) in this case equals

$$
\mathcal{B}=C^{\infty}(N) \otimes \Lambda V^{*} \otimes \Lambda V
$$

This algebra has a $\mathbf{Z} \times \mathbf{Z}$-grading induced by the two Grassmann algebras. The grading on $\Lambda V^{*}$ is called the ghost number grading, the grading on $\Lambda V$ is called the anti-ghost number grading. Subtracting the two gives the total ghost number grading. So elements of $\mathcal{B}^{p, q}=C^{\infty}(N) \otimes \Lambda^{p} V^{*} \otimes \Lambda^{q} V$ are elements of total ghost number $p-q$. Actually, in the group case, $\mathcal{B}$ is a double complex with two differentials $\delta$ and $d$, both of degree +1 . We shall define them now in general. Let $v_{\alpha}$ be a basis of $V$ such that $\left(F, v_{\alpha}\right)=f_{\alpha}$. The parentheses denote the natural pairing between $V$ and its linear dual. As elements of $\Lambda^{1} V$, they have degree -1 and generate $\Lambda V$. We introduce also generators $\eta^{\alpha}$ of $\Lambda V^{*}$, having degree +1 and being dual to the $v_{\alpha}$. Note that $\mathcal{B}$ is generated as an algebra by the functions and the $2 m$ elements $v_{\alpha}$ and $\eta^{\alpha}$. We define

$$
\begin{array}{ll}
\delta(f)=0, & d(f)=\left\{f_{\alpha}, f\right\} \otimes \eta^{\alpha} \otimes 1+\text { "more", } \\
\delta\left(\eta^{\alpha}\right)=0, & d\left(\eta^{\alpha}\right)=-\frac{1}{2} c_{\beta \gamma}^{\alpha} \otimes \eta^{\beta} \wedge \eta^{\gamma} \otimes 1+\text { "more" } \\
\delta\left(v_{\alpha}\right)=f_{\alpha} \otimes 1 \otimes 1, & d\left(v_{\alpha}\right)=-c_{\alpha \beta}^{\gamma} \otimes \eta^{\beta} \otimes v_{\gamma}+\text { "more", }
\end{array}
$$

where $f \in C^{\infty}(N)$ and "more" means that there are terms with more ghost-variables involved, such that $d^{2}=\delta d+d \delta$. These higher order terms only vanish in the case where the structure functions $c_{\alpha \beta}^{\gamma}$ are constants. In general, they involve so-called higher order structure functions, who are defined inductively. It is obvious that $\delta^{2}=0$. In fact the subcomplex $\left(\Lambda V \otimes C^{\infty}(N), \delta\right)$ is just the Koszul resolution of $C^{\infty}(N) / I$. As was observed by Stasheff ([S]), also in the non-regular case BRST theory involves a resolution of this quotient ring. The total operator $D=\delta+d$ is called the BRST operator. We will now give a very nice formulation of this differential in terms of super Poisson algebras and state a beautiful theorem on the existence of a BRST charge.

Recall that a super Poisson algebra is an algebra with a $\mathbf{Z}_{2}$-grading and a super Lie algebra structure that is compatible with the ring structure in the sense that a graded Leibnitz rule holds:

$$
\left\{a_{1} \cdot a_{2}, a_{3}\right\}=a_{1} \cdot\left\{a_{2}, a_{3}\right\}+(-1)^{\operatorname{deg}\left(a_{2}\right) \cdot \operatorname{deg}\left(a_{1}\right)} a_{2} \cdot\left\{a_{1}, a_{3}\right\} .
$$

For odd elements the Poisson bracket is commutative, for any other elements with a definite grading the Poisson bracket is anti-commutative. Let us introduce a super Poisson structure on $\mathcal{B}$ that extends the one on $C^{\infty}(N)$. Besides the bracket between 
two functions the only non-vanishing bracket between generators is $\left\{v_{\alpha}, \eta^{\beta}\right\}=$ $\left\{\eta^{\beta}, v_{\alpha}\right\}=\delta_{\alpha}^{\beta}$. This determines the Poisson bracket completely using the Leibnitz rule. Henneaux proved the following theorem $([\mathrm{H}])$ of Fradkin and Fradkina and others.

Theorem 1. Suppose $0 \in V^{*}$ is a regular value of $F$. Then there exists an element $Q \in \mathcal{B}$, the BRST charge, of total degree 1 such that

(i) $\{Q, Q\}=0$,

(ii) $Q^{(0)}=f_{\alpha} \otimes \eta^{\alpha} \otimes 1$,

where $Q^{(i)}$ denotes the component of $Q$ in $\mathcal{B}^{i+1, i}$. This $Q$ is, up to super canonical transformations, uniquely associated to the constraint manifold $Z$.

Using this BRST charge we can define a differential $D$ on $\mathcal{B}, D=\{Q, \cdot\}$. As the notation already suggests, this differential is the same as the one defined above. Although the formulation in terms of a Poisson algebra is both natural and transparent it does not help very much for computing the cohomology. It only helps to see that the cohomology space $H_{D}^{\bullet}(\mathcal{B})$ inherits a Poisson structure. Nevertheless, the cohomology of $D$ is completely known and was first computed in [H-T].

To explain a little bit of this work, write $D$ as the sum of the two derivations defined earlier. The differential $\delta$ has, because of the regularity of the $f_{\alpha}$, only non-zero cohomology in $\mathcal{B}^{p, 0}$, i.e, anti-ghost degree zero. Therefore, the spectral sequence, associated to this complex $\left(\mathcal{B}^{p, q}, \delta, d\right)$ is degenerated, and thus

$$
H_{D}^{\bullet}(\mathcal{B})=H_{d}^{\bullet}\left(H_{\delta}(\mathcal{B})\right) \text {. }
$$

Using the fact that $C^{\infty}(N) / I \simeq C^{\infty}(Z)$ it is easy to compute that $H_{\delta}(\mathcal{B}) \simeq$ $C^{\infty}(Z) \otimes \Lambda V^{*}$. This algebra with differential $d$ equals the complex of differential forms on the vertical tangent bundle of $Z$ (this is the subbundle mentioned above). Therefore the BRST cohomology for regular first class constraints equals the vertical cohomology of the constraint manifold.

In the case $0 \in V^{*}$ is not a regular value of $F$, but only weakly regular, i.e., $Z$ is a submanifold but its codimension is smaller than the number of constraints, there is a similar theorem on the existence of a BRST charge ([B-V, F-H-S-T]). In this case there are relations among the constraints and the differential $\delta$ does not give a resolution anymore. The constraints are called reducible. One enlarges the algebra $\mathcal{B}$ (introducing "ghosts for ghosts") and modifies $\delta$ and $d$ such that $\delta$ becomes a resolution of $C^{\infty}(N) / I$ (Koszul-Tate resolution) and $d$ gives the vertical cohomology again.

In the next sections we shall neglect $\delta$ and work directly with the constraint manifold $Z$. Only considering positive ghost degree is common. The negative degrees are only added to obtain a resolution for $C^{\infty}(N) / I . \delta$ is always constructed in such a way that the associated spectral sequence degenerates. In fact in the next sections we will always assume that the symplectic manifold $N$ is a cotangent bundle $T^{*} M$ and that the constraints come from Hamiltonian $G$-actions that are induced by arbitrary actions on $M . M$ is called the configuration space. $Z$ is then a subbundle of the vector bundle $N$ over $M$ and the reduced phase space $X=Z / G$ equals $T^{*}(M / G)$. In the sequel we shall use $M$ instead of $Z$, because the action on $Z$ comes from the one on $M$. It is in this way that we study the BRST complex associated to an arbitrary Lie group action on an arbitrary manifold. The new element is that $G$ need not be finite dimensional and that for transitive $G$ actions 
we obtain the model for topological field theories that we were looking for. For later convenience, we already mention that the positive degree part of (2) can also be written as $\operatorname{Hom}_{\mathbb{R}}\left(\wedge V, C^{\infty}(N)\right)$.

As the manipulation of infinite dimensional geometrical objects is tricky, we will work in an algebraic framework. We will start by a mathematical description of the situation in which we will work. Then, we will recall the algebraic structure on which our construction relies, namely the crossed product structure.

\section{Algebraic Set-up}

3.1 Presentation. Let $G$ be a Lie group (not necessarily finite dimensional) and let $\mathrm{g}$ be its Lie algebra. Let $M$ be a differentiable paracompact manifold of dimension $n$. We assume that $G$ acts on $M$ in such a way that we have a $C^{\infty}$-morphism from $G$ to $\operatorname{Diff}(M)$. By differentiating this homomorphism at the unity, we get a Lie algebra morphism $\sim: \mathfrak{g} \rightarrow \Gamma(M, T M)$. Let $W$ be the trivial vector bundle $M \times \mathfrak{g}$ and let $T_{v}(M)$ be the image of the following vector bundle map:

$$
\begin{aligned}
\phi: M \times \mathfrak{g} & \rightarrow T M \\
(m, X) & \mapsto(m, \tilde{X}(m)) .
\end{aligned}
$$

All along this paper, we assume that $T_{v}(M)$ is a fiber bundle. The following lemma provides a criterion for this assumption to be satisfied.

Lemma 3.1.1. $T_{v}(M)$ is a fiber bundle if and only if for any $m$ in $M$, the subspace $\{\tilde{X}(m) \mid X \in \mathfrak{g}\}$ has the same dimension.

Proof of Lemma 3.1.1. Let $m$ be in $M$. Put $\mathfrak{g}_{m}=\{X \in \mathfrak{g} \mid \tilde{X}(m)=0\}$. Let $\mathfrak{f}$ be a complement of $\mathfrak{g}_{m}$ in $\mathfrak{g}$. There exists a neighborhood of $m$ such that the map

$$
\begin{aligned}
U \times \mathfrak{l} & \rightarrow T M \\
(m, X) & \mapsto(m, \tilde{X}(m)) .
\end{aligned}
$$

has a constant rank and is injective. This proves that $T_{v}(M)$ is a vector bundle. The converse is obvious.

We will also denote by $\phi$ the vector bundle map from $W$ to $T_{v}(M)$. Let $U$ be an open subset of $M$. We will write $\Phi(U)$ for the morphism from $\Gamma(U, W)$ to $\Gamma\left(U, T_{v}(M)\right)$. For simplicity, $\Phi(M)$ will be denoted by $\Phi$. Moreover, we put $A=C^{\infty}(M)$.

Lemma 3.1.2. The A-module morphism $\Phi: \Gamma(M, W) \rightarrow \Gamma\left(M, T_{v}(M)\right)$ is onto.

Proof of Lemma 3.1.2. Let $\left(X_{i}\right)_{i \in I}$ be a basis of $\mathfrak{g}$ and let $m$ be a point in $M$. Then $\left(\tilde{X}_{i}(m)\right)_{i \in I}$ is a generating system of $\left(T_{v}(M)\right)_{m}$. We extract a basis $\left(\tilde{X}_{1}(m), \ldots, \tilde{X}_{p}(m)\right)$ from it. Let $\mathcal{V}_{m}$ be a neighborhood of $m$ such that for all $y$ in $\mathcal{V}_{m},\left(\tilde{X}_{1}(y), \ldots, \tilde{X}_{p}(y)\right)$ is a basis of $\left(T_{v}(M)\right)_{y}$. Then, let $\mu$ in $\Gamma\left(\mathcal{V}_{m}, T_{v}(M)\right)$. There exists $\left(f_{i}\right)_{i \in[1, p]}$ in $C^{\infty}\left(\mathcal{V}_{m}\right)$ such that $\mu=\sum_{l=1}^{p} f_{i} \tilde{X}_{i}$. This means $\mu=\Phi\left(\mathcal{V}_{m}\right)\left(\sum_{i=1}^{p} f_{i} X_{i}\right)$. So $\Phi\left(\mathcal{V}_{m}\right)$ is onto.

Let $\left(\mathcal{U}_{j}\right)_{j \in J}$ be a locally finite refinement of $\left(\mathcal{V}_{m}\right)_{m \in M}$. Then, $\Phi\left(\mathcal{U}_{j}\right)$ is also onto (because any element of $\Gamma\left(\mathcal{U}_{j}, T_{v}(M)\right)$ can be written $\left.\sum_{i=1}^{p} f_{i} \tilde{X}_{i}\right)$. 
Let $\tau$ be in $\Gamma\left(M, T_{v}(M)\right)$ and let $\left(\alpha_{j}\right)_{j \in J}$ be a partition of unity subordinate to $\mathcal{U}_{j}$. Then, there exists $\sigma_{j}$ in $\Gamma\left(\mathcal{U}_{j}, W\right)$ such that $\left.\tau\right|_{\mathcal{U}_{j}}=\Phi\left(\mathcal{U}_{j}\right)\left(\sigma_{j}\right)$. We have $\Phi\left(\sum_{j=1}^{p} \alpha_{j} \sigma_{j}\right)=\tau$. This finishes the proof of Lemma 3.1.2.

If $\mathfrak{g}$ is not finite dimensional, $\Gamma(M, W)$ contains strictly $A \otimes \mathfrak{g}$. Throughout this paper, we will make the following hypothesis on our action. We assume that the restriction of $\Phi$ to $A \otimes \mathfrak{g}$ is also onto. So we have the following exact sequence:

$$
\{0\} \rightarrow P \rightarrow A \otimes \mathfrak{g} \stackrel{\Phi}{\rightarrow} \Gamma\left(M, T_{v}(M)\right) \rightarrow\{0\} .
$$

The statement of the previous lemma says that this hypothesis is always satisfied if $\mathfrak{g}$ is finite dimensional. The proof of the same lemma shows that it is also always satisfied if $M$ is compact. If $M$ is not necessarily compact, this assumption is not always satisfied. The group of diffeomorphisms with compact support provides a counterexample. Nevertheless, this hypothesis is always satisfied for $\operatorname{Diff}(M)$ which is the example we will be the most interested in. Indeed, one can lift any vector field to the constant section equal to the considered vector field. The above hypothesis will allow us to make the BRST cohomology appear as a modification of a Lie algebra cohomology.

Using this assumption and the fact that $\Gamma\left(M, T_{v} M\right)$ is a projective $A$-module ([Hu], p. 31, [W], p. 100), we conclude that there exists a $A$-module $R$ such that $P \oplus R=A \otimes \mathrm{g}$. In the case where the group acting is $\operatorname{Diff}(M)$, we can take for $R$ the $A$-module $\Gamma(M, T M)$ (which has to be seen as constant sections). The decomposition of an element of $\sigma$ of $A \otimes \mathfrak{g}$ is in this case $\sigma=(\sigma-\Phi(\sigma))+\Phi(\sigma)$.

3.2 Crossed Product Structure on $A \otimes \mathfrak{g}$. Let us first recall what a crossed product is $([\mathrm{F}, \mathrm{M}])$. In this paragraph $A$ is any $\mathbb{R}$-algebra (commutative associative with unity) and $\mathrm{g}$ is any $\mathbb{R}$-Lie algebra. Assume that there exists a Lie algebra morphism $\sigma: \mathfrak{g} \rightarrow \operatorname{Der}(A)$. Put $\mathcal{L}=A \otimes \mathfrak{g}$. Then $\mathcal{L}$ is a $A$-module. The following bracket

$$
\begin{aligned}
& \forall(a, b) \in A, \forall(X, Y) \in \mathfrak{g}, \\
& \quad[a \otimes X, b \otimes Y]=a \sigma(X)(b) Y-b \sigma(Y)(a) X+a b \otimes[X, Y]
\end{aligned}
$$

endows $\mathcal{L}$ with a Lie bracket structure. Moreover, we have a compatibility relation between these two structures. Namely,

$$
\begin{aligned}
& \forall(a \otimes X, b \otimes X) \in \mathcal{L}^{2}, \forall f \in A, \\
& {[a \otimes X, f(b \otimes Y)]=f[a \otimes X, b \otimes Y]+a \sigma(X)(f)^{b} \otimes Y .}
\end{aligned}
$$

One says that $\mathcal{L}$ is the crossed product of $A$ and $\mathfrak{g}$.

Definition . With the same notations as above, we will say that $V$ is a $A$-gmodule if

- $V$ is a A-module

- $V$ is a g-module

- These two structures are compatible in the following sense:

$$
\forall a \in A, \forall X \in \mathfrak{g}, \forall v \in V, X \cdot(a \cdot v)-a \cdot(X \cdot v)=\sigma(X)(a) v .
$$




\section{Remarks.}

1) If $V$ is a $A-\mathfrak{g}$-module then $\operatorname{Hom}_{A}(V, A)$ endowed with the two following operations

$$
\begin{gathered}
\forall X \in \mathfrak{g}, \forall a \in A, \forall \mathfrak{g} \in \operatorname{Hom}_{A}(V, A), \forall v \in V, \\
(X \cdot g)(v)=\sigma(X)[g(v)]-g(X \cdot v) \\
(a \cdot g)(v)=a g(v)
\end{gathered}
$$

is a $A-\mathfrak{g}$-module.

2) If $V$ is a $A-\mathfrak{g}$-module, $S_{A}^{p}(V)$ has a natural $A-\mathfrak{g}$-module structure ( $\mathfrak{g}$ acts on $S_{A}^{p}(V)$ by derivation).

We come back to our situation: $A=C^{\infty}(M), g$ is the Lie algebra of a Lie group $G$ acting on $M$. We endow $A \otimes \mathfrak{g}$ with a crossed product structure using the Lie algebra morphism $\sim: \mathfrak{g} \rightarrow \operatorname{Der}(A)$. Remember that $\Gamma\left(M, T_{v}(M)\right)$ has a natural $A$-module structure and also a Lie algebra structure (given by the usual bracket of vector fields). The morphism $\Phi: A \otimes \mathfrak{g} \rightarrow \Gamma\left(M, T_{v}(M)\right)$ given by $\Phi(f \otimes X)=f \tilde{X}$ is a Lie algebra morphism and a $A$-module morphism. Hence, the kernel of $\Phi, P$, inherits a $A-\mathfrak{g}$-module structure. Here, the action of $\mathfrak{g}$ on $P$ is given by the Lie bracket (6) in $A \otimes \mathfrak{g}$ :

$$
\forall X \in \mathfrak{g}, \forall p \in P, \quad X \cdot p=[1 \otimes X, p] .
$$

From the previous remarks, we deduce that $\left(S_{A}^{p}(P)\right)^{*}=\operatorname{Hom}_{A}\left(S_{A}^{p}(P), A\right)$ has a natural $A-\mathfrak{g}$-module structure.

\section{The BRST Complex}

We are now about to define the BRST complex for the situation we are interested in. Let $M$ be the configuration space of some physical system and let $G$ act on $M$ as a symmetry group for this system. If $G$ acts freely on $M$, then $P=\{0\}$ in (5) and the BRST algebra is $\mathcal{S}=\operatorname{Hom}_{\mathbb{R}}(\bigwedge \mathfrak{g}, A)=\operatorname{Hom}_{A}\left(\bigwedge_{A}(A \otimes \mathfrak{g}), A\right)$. In the case where the action is not free but $T_{v} M$ is still a vector bundle, we have to add ghosts for ghosts that are commuting to define the BRST algebra $\mathcal{S}$. For simplicity, we put $F=A \otimes g$. Then, the BRST algebra is

$$
\mathcal{S}=\bigoplus_{p, q} \operatorname{Hom}_{A}\left(\bigwedge_{A}^{p} F,\left(S_{A}^{q}(P)\right)^{*}\right) \simeq \bigoplus_{p, q} \operatorname{Hom}_{A}\left(\bigwedge_{A}^{p} F, \underset{A}{\otimes} S_{A}^{q}(P), A\right) .
$$

Moreover, an element of $\bigoplus_{p, q} \operatorname{Hom}_{A}\left(\bigwedge_{A}^{p} F,\left(S_{A}^{q}(P)\right)^{*}\right)$ has grading $p+2 q$. Let us now describe the differential $D$ in $\mathcal{S}$. Let $\Omega$ be an element of $\operatorname{Hom}_{A}\left(\bigwedge_{A}^{p} F\right.$, $\left.\left(S_{A}^{q}(P)\right)^{*}\right)$. Then, $\quad D \Omega=D_{1} \Omega+D_{2} \Omega \quad$ with $\quad D_{1} \Omega \in \operatorname{Hom}_{A}\left(\bigwedge_{A}^{p+1} F,\left(S_{A}^{q}(P)\right)^{*}\right)$ and $D_{2} \Omega \in \operatorname{Hom}_{A}\left(\bigwedge_{A}^{p-1} F,\left(S_{A}^{q+1}(P)\right)^{*}\right)$. For all $\left(\mathcal{X}_{1}, \ldots, \mathcal{X}_{p+1}\right)$ in $F$ and $\left(\mathcal{H}_{1}, \ldots, \mathcal{H}_{q+1}\right)$ in $P$, we have

$$
\begin{aligned}
D_{1} \Omega\left(\mathcal{X}_{1}, \ldots, \mathcal{X}_{p+1}\right)= & \sum_{i}(-1)^{i-1} \mathcal{X}_{i} \cdot\left[\Omega\left(\mathcal{X}_{1}, \ldots, \widehat{\mathcal{X}}_{i}, \ldots, \mathcal{X}_{p+1}\right)\right] \\
& +\sum_{i<j} \Omega\left(\left[\mathcal{X}_{i}, \mathcal{X}_{j}\right], \mathcal{X}_{1}, \ldots, \widehat{\mathcal{X}}_{i}, \ldots, \widehat{\mathcal{X}}_{j}, \ldots \mathcal{X}_{p+1}\right)(-1)^{i+j}
\end{aligned}
$$


and

$$
\begin{aligned}
& D_{2} \Omega\left(\mathcal{X}_{1}, \ldots, \mathcal{X}_{p-1}\right)\left(\mathcal{H}_{1}, \ldots, \mathcal{H}_{q+1}\right) \\
& \quad=\sum_{i} \Omega\left(\mathcal{H}_{i}, \mathcal{X}_{1}, \ldots, \mathcal{X}_{p-1}\right)\left(\mathcal{H}_{1}, \ldots, \widehat{\mathcal{H}}_{i}, \ldots, \mathcal{H}_{p+1}\right) .
\end{aligned}
$$

If we identify $\mathcal{S}$ with $\bigoplus_{p, q} \operatorname{Hom}_{\mathbb{R}}\left(\bigwedge_{\mathbb{R}}^{p} \mathfrak{g},\left(S_{A}^{q}(P)\right)^{*}\right)$, then $D_{1}$ is nothing but the differential of the standard complex for the computation of the cohomology of $\mathfrak{g}$ with values $S_{A}(P)^{*}$ (see [F] p. 136). Note that here, we define the cohomology of $\mathrm{g}$ as in $[\mathrm{H}-\mathrm{S}]$, that is to say without taking into account its topology. We will denote by $\mathcal{C}(G)$ - or more simply $\mathcal{C}$ if there is no ambiguity - the BRST complex associated to the action of $G$. We have $\mathcal{C}(G)=(\mathcal{S}, D)$. We are now going to exhibit a double complex structure on $\mathcal{C}$. On the algebra $\mathcal{S}$, we define two degrees whose sum will give our initial degree. The first degree of an element of $\operatorname{Hom}_{A}\left(\bigwedge_{A}^{p} F,\left(S_{A}^{q}(P)\right)^{*}\right)$ is $q$ and its second degree is $p+q$. The first (respectively second) degree is preserved by $D_{1}$ (respectively $D_{2}$ ) and is increased by one by $D_{2}$ (respectively $D_{1}$ ).

Proposition 4.0.1. The two gradings defined above and the differentials $D_{1}, D_{2}$ define a double complex structure on $\mathcal{S}$ whose associated total complex is the BRST complex $\mathcal{C}(G)$.

Proof of Proposition 4.0.1. We have to prove the following relations $D_{1}^{2}=0, D_{2}^{2}=$ $0, D_{1} D_{2}=-D_{2} D_{1}$. It is a standard computation to establish the first one. Let us prove the second one. Let $\Omega$ be in $\operatorname{Hom}_{A}\left(\bigwedge_{A}^{p} F,\left(S_{A}^{q}(P)\right)^{*}\right)$. For all $\left(\mathcal{X}_{1}, \ldots, \mathcal{X}_{p-2}\right)$ in $F$ and all $\left(\mathcal{H}_{1}, \ldots, \mathcal{H}_{q+2}\right)$ in $P$, we have

$$
\begin{aligned}
& D_{2}^{2} \Omega\left(\mathcal{X}_{1}, \ldots, \mathcal{X}_{p-2}\right)\left(\mathcal{H}_{1}, \ldots, \mathcal{H}_{q+2}\right) \\
& =\sum_{i, j} \Omega\left(\mathcal{H}_{i}, \mathcal{H}_{j}, \mathcal{X}_{1}, \ldots, \mathcal{X}_{p-2}\right)\left(\mathcal{H}_{1}, \ldots, \widehat{\mathcal{H}}_{i}, \ldots, \widehat{\mathcal{H}}_{j}, \ldots \mathcal{H}_{q+2}\right)=0,
\end{aligned}
$$

using the fact that this expression is symmetric in the $\mathcal{H}_{i}$. Let us now check the third relation. We compute separately $D_{1} D_{2}$ and $D_{2} D_{1}$. They are both in $\operatorname{Hom}_{A}\left(\bigwedge_{A}^{p} F,\left(S_{A}^{q+1}(P)\right)^{*}\right)$. Keeping the same notations, on one hand we have

$$
\begin{aligned}
& D_{1} D_{2} \Omega\left(\mathcal{X}_{1}, \ldots, \mathcal{X}_{p}\right)\left(\mathcal{H}_{1}, \ldots, \mathcal{H}_{q+1}\right) \\
& =\sum_{i}(-1)^{i-1}\left(\mathcal{X}_{i}, \cdot\left(D_{2} \Omega\left(\mathcal{X}_{1}, \ldots, \widehat{\mathcal{X}}_{i}, \ldots, \mathcal{X}_{p}\right)\right)\right)\left(\mathcal{H}_{1}, \ldots, \mathcal{H}_{q+1}\right) \\
& \quad+\sum_{i<j} D_{2} \Omega\left(\left[\mathcal{X}_{i}, \mathcal{X}_{j}\right], \ldots, \widehat{\mathcal{X}}_{i}, \ldots, \widehat{\mathcal{X}}_{j}, \ldots \mathcal{X}_{p}\right)\left(\mathcal{H}_{1}, \ldots, \mathcal{H}_{q}\right)(-1)^{i+j} \\
& =\sum_{i, k} \Phi\left(\mathcal{X}_{i}\right)\left(\Omega\left(\mathcal{H}_{k}, \mathcal{X}_{i}, \ldots, \widehat{\mathcal{X}}_{i}, \ldots, \mathcal{X}_{p}\right)\left(\mathcal{H}_{1}, \ldots, \widehat{\mathcal{H}}_{k}, \ldots, \mathcal{H}_{q+1}\right)\right)(-1)^{i-1} \\
& \quad-\sum_{i, k} D_{2} \Omega\left(\mathcal{X}_{1}, \ldots, \widehat{\mathcal{X}}_{i}, \ldots, \mathcal{X}_{p}\right)\left(\mathcal{H}_{1}, \ldots,\left[\mathcal{X}_{i}, \mathcal{H}_{k}\right] \ldots, \mathcal{H}_{q+1}\right)(-1)^{i-1} \\
& \quad+\sum_{i<j, k} \Omega\left(\mathcal{H}_{k},\left[\mathcal{X}_{i}, \mathcal{X}_{j}\right], \mathcal{X}_{1}, \ldots, \widehat{\mathcal{X}}_{i}, \ldots, \widehat{\mathcal{X}}_{j}, \ldots, \mathcal{X}_{p}\right) \\
& \quad \times\left(\mathcal{H}_{1}, \ldots, \widehat{\mathcal{H}}_{k}, \ldots, \mathcal{H}_{q+1}\right)(-1)^{i+j}
\end{aligned}
$$




$$
\begin{aligned}
= & \sum_{i, k} \Phi\left(\mathcal{X}_{i}\right)\left(\Omega\left(\mathcal{H}_{k}, \mathcal{X}_{i}, \ldots, \widehat{\mathcal{X}}_{i}, \ldots, \mathcal{X}_{p}\right)\left(\mathcal{H}_{1}, \ldots, \widehat{\mathcal{H}}_{k}, \ldots, \mathcal{H}_{q+1}\right)\right)(-1)^{i-1} \\
& -\sum_{i, k} \Omega\left(\left[\mathcal{X}_{i}, \mathcal{H}_{k}\right], \mathcal{X}_{1}, \ldots, \widehat{\mathcal{X}}_{i}, \ldots, \mathcal{X}_{p}\right)\left(\mathcal{H}_{1}, \ldots, \widehat{\mathcal{H}}_{k} \ldots, \mathcal{H}_{q+1}\right)(-1)^{i-1} \\
& \left.-\sum_{i, j \neq k} \Omega\left(\mathcal{H}_{j}, \mathcal{X}_{1}, \ldots, \widehat{\mathcal{X}}_{i}, \ldots, \mathcal{X}_{p}\right) \mathcal{H}_{1}, \ldots, \widehat{\mathcal{H}}_{j}, \ldots,\left[\mathcal{X}_{i}, \mathcal{H}_{k}\right], \ldots, \mathcal{H}_{q+1}\right)(-1)^{i-1} \\
& +\sum_{i<j} \Omega\left(\mathcal{H}_{k},\left[\mathcal{X}_{i}, \mathcal{X}_{j}\right], \mathcal{X}_{1}, \ldots, \widehat{\mathcal{X}}_{i}, \ldots, \widehat{\mathcal{X}}_{j}, \ldots, \mathcal{X}_{p}\right) \\
& \times\left(\mathcal{H}_{1}, \ldots, \widehat{\mathcal{H}}_{k}, \ldots, \mathcal{H}_{q+1}\right)(-1)^{i+j} .
\end{aligned}
$$

On the other hand,

$$
\begin{aligned}
& D_{2} D_{1} \Omega\left(\mathcal{X}_{1}, \ldots, \mathcal{X}_{p}\right)\left(\mathcal{H}_{1}, \ldots, \mathcal{H}_{q}\right)=D_{1} \Omega\left(\mathcal{H}_{k}, \mathcal{X}_{1}, \ldots, \mathcal{X}_{p}\right)\left(\mathcal{H}_{1}, \ldots, \widehat{\mathcal{H}}_{k}, \ldots, \mathcal{H}_{q}\right) \\
& =\sum_{i, k}\left(\mathcal{X}_{i} \cdot\left(\Omega\left(\mathcal{H}_{k}, \mathcal{X}_{1}, \ldots, \widehat{\mathcal{X}}_{i}, \ldots, \mathcal{X}_{p}\right)\right)\right)\left(\mathcal{H}_{1}, \ldots, \widehat{\mathcal{H}}_{k}, \ldots, \mathcal{H}_{q+1}\right)(-1)^{i} \\
& \quad+\sum_{k} \mathcal{H}_{k} \cdot\left(\Omega\left(\mathcal{X}_{1}, \ldots, \mathcal{X}_{p}\right)\right)\left(\mathcal{H}_{1}, \ldots, \widehat{\mathcal{H}}_{k}, \ldots, \mathcal{H}_{q+1}\right) \\
& \quad+\sum_{k, i<j} \Omega\left(\left[\mathcal{X}_{i}, \mathcal{X}_{j}\right], \mathcal{H}_{k}, \mathcal{X}_{1}, \ldots, \widehat{\mathcal{X}}_{i}, \ldots, \widehat{\mathcal{X}}_{j}, \ldots, \mathcal{X}_{p}\right) \\
& \quad \times\left(\mathcal{H}_{1}, \ldots, \widehat{\mathcal{H}}_{k}, \ldots, \mathcal{H}_{q+1}\right)(-1)^{i+j} \\
& \quad+\sum_{k, i} \Omega\left(\left[\mathcal{H}_{k}, \mathcal{X}_{i}\right], \mathcal{X}_{1}, \ldots, \widehat{\mathcal{X}}_{i}, \ldots, \mathcal{X}_{p}\right)\left(\mathcal{H}_{1}, \ldots, \widehat{\mathcal{H}}_{k}, \ldots, \mathcal{H}_{q+1}\right)(-1)^{i}
\end{aligned}
$$

As the second term equals zero, it is clear that $D_{1} D_{2}=-D_{2} D_{1}$.

\section{Cohomology of the BRST Complex}

In this section, we are going to compute the cohomology of the BRST complex by using its double complex structure. Let $\Gamma\left(M, \bigwedge T_{v}(M)^{*}\right)$ be the space of vertical differential forms. We endow it with the natural grading of differential forms and with the de Rham differential $d$.

Theorem 5.0.1. Let $\mathcal{E}$ be the map from $\Gamma\left(M, \bigwedge T_{v}(M)^{*}\right)$ to $\bigoplus_{p, q} \operatorname{Hom}_{A}\left(\bigwedge_{A}^{p} F\right.$, $\left.\left(S_{A}^{q}(P)\right)^{*}\right)$ defined as follows. For all $\omega$ in $\Gamma\left(M, \wedge^{p} T_{v}(M)^{*}\right), \mathcal{E}(\omega)$ is the element of $\bigoplus_{q} \operatorname{Hom}_{A}\left(\bigwedge_{A}^{p} F,\left(S_{A}^{q}(P)\right)^{*}\right)$ determined by

$$
\forall\left(\mathcal{X}_{1}, \ldots, \mathcal{X}_{p}\right) \in F, \quad \mathcal{E}(\omega)\left(\mathcal{X}_{1}, \ldots, \mathcal{X}_{p}\right)=\omega\left(\Phi\left(\mathcal{X}_{1}\right), \ldots, \Phi\left(\mathcal{X}_{p}\right)\right)
$$

$\mathcal{E}$ is a quasi-isomorphism between the complex $\left(\Gamma\left(M, \bigwedge T_{v}(M)^{*}\right), d\right)$ and the BRST complex $\mathcal{C}$.

This theorem has been proved in a more general but finite dimensional case by [F-H-S-T], although there is a slight difference. In their set-up $P$ is a free module, whereas here it is only projective.

Proof of Theorem 5.0.1. A small computation shows that $\mathcal{E}$ is a morphism of complexes. Moreover, $\mathcal{E}$ is injective because $\Phi$ is onto. It is easy to see that 


$$
\operatorname{Im} \mathcal{E} \simeq \bigoplus_{p} \operatorname{Hom}_{A}\left(\bigwedge_{A}^{p}(F / P), A\right) \simeq \operatorname{Hom}_{A}\left(\bigwedge \Gamma\left(M, T_{v} M\right), A\right) \simeq \Gamma\left(M, \bigwedge T_{v} M^{*}\right)
$$

Let $\mathcal{I}$ denote the subcomplex $\left(\operatorname{Im} \mathcal{E}, D=D_{1}\right)$ of $\mathcal{C}$. It is clear that $\mathcal{I}$ is isomorphic to $\Gamma\left(M, \bigwedge T_{v} M^{*}\right)$. Let us show that the natural injection from $\mathcal{I}$ to $\mathcal{C}$ is a quasiisomorphism. For this purpose, we are going to use spectral sequences. We first compute the cohomology of $\mathcal{C}$ with respect to $D_{2}$. Let $\mathcal{J}$ be the following complex:

$$
\ldots \rightarrow \bigoplus_{p} \operatorname{Hom}_{A}\left(\bigwedge_{A}^{p} F,\left(S_{A}^{q}(P)\right)^{*}\right) \stackrel{D_{2}}{\rightarrow} \bigoplus_{p} \operatorname{Hom}_{A}\left(\bigwedge_{A}^{p} F,\left(S_{A}^{q+1}(P)\right)^{*}\right) \rightarrow \ldots
$$

It is clear that $\left.H^{0}(\mathcal{J})=\bigoplus_{p} \operatorname{Hom}_{A}\left(\bigwedge_{A}^{p} F / P\right), A\right)$. Let us show that for $q>0$, $H^{q}(\mathcal{J})=\{0\}$. For this, we will use the decomposition $P \oplus R=F$. Let $q>0$. The cycles of $\bigoplus_{p} \operatorname{Hom}_{A}\left(\bigwedge_{A}^{p} F,\left(S_{A}^{q}(P)\right)^{*}\right)$ are the elements of $\bigoplus_{p} \operatorname{Hom}_{A}\left(\bigwedge_{A}^{p}(F / P)\right.$, $\left.\left(S_{A}^{q}(P)\right)^{*}\right)$. Let us now prove that all the elements of $\bigoplus_{p} \operatorname{Hom}_{A}\left(\bigwedge_{A}^{p}(F / P)\right.$, $\left.\left(S_{A}^{q}(P)\right)^{*}\right)$ are boundaries. Let $\omega$ be in $\bigoplus_{p} \operatorname{Hom}_{A}\left(\bigwedge_{A}^{p}(F / P),\left(S_{A}^{q}(P)\right)^{*}\right)$. We can see $\omega$ as a map from $(\stackrel{p}{\otimes} F) \otimes(\stackrel{q}{\otimes} P)$ to $A$ which is antisymmetric in the first $p$ variables and symmetric in the $q$ last variables. Using the decomposition $P \oplus R=F$, we extend $\omega$ into a $p+q$-multilinear map $\tilde{\omega}$ from $\stackrel{p+1}{\otimes} F \otimes \stackrel{q-1}{\otimes} P$. The map $\tilde{\omega}$ is symmetric in the last $q-1$ variable. By a standard process, we transform $\tilde{\omega}$ into a $p+q$-multilinear map $\Omega$ which is also going to be antisymmetric in the $p+1$ first variables. For all $\left(\mathcal{X}_{1}, \ldots, \mathcal{X}_{p+1}\right)$ in $F$ and all $\left(\mathcal{H}_{1}, \ldots, \mathcal{H}_{q-1}\right)$ in $P$, we put

$$
\begin{aligned}
\Omega\left(\mathcal{X}_{1}, \ldots, \mathcal{X}_{p+1}\right)\left(\mathcal{H}_{1}, \ldots, \mathcal{H}_{q-1}\right)= & \sum_{\sigma \in \sum p+1}(-1)^{\varepsilon(\sigma)} \tilde{\omega}\left(\mathcal{X}_{\sigma(1)}, \ldots, \mathcal{X}_{\sigma(p+1)}\right) \\
& \times\left(\mathcal{H}_{1}, \ldots, \mathcal{H}_{q-1}\right)
\end{aligned}
$$

Then $\Omega$ belongs to $\operatorname{Hom}_{A}\left(\bigwedge_{A}^{p+1} F,\left(S_{A}^{q}(P)\right)^{*}\right)$. Moreover $D_{2} \Omega=\omega$. So we have proved that $H^{q}(\mathcal{J})=\{0\}$. Now, using a double complex argument, it is clear that $H^{\bullet}\left(\mathcal{I}, D=D_{1}\right)=H^{\bullet}(\mathcal{C}, D)$. As we have already noticed that the complex $\mathcal{I}$ was isomorphic to $\Gamma\left(M, \bigwedge T_{v} M^{*}\right)$, this finishes the proof of Theorem 5.0.1.

\section{BRST Cohomology and Equivariant Cohomology}

In this section, we apply the results of the previous one to a very special situation, so as to obtain the BRST differential algebra for topological theories. We start with some recollections.

6.1 The Weil Algebra. Let $\mathfrak{h}$ be a finite dimensional real Lie algebra. Let us recall ([C]) that its Weil algebra is $W(\mathfrak{h})=\wedge\left(\mathfrak{h}^{*}\right) \otimes S\left(\mathfrak{h}^{*}\right)$. The grading on $W(\mathfrak{h})$ is as follows:

- The elements of $\wedge^{1}\left(\mathfrak{h}^{*}\right)$ have degree 1 ,

- The elements of $S^{1}\left(\mathfrak{h}^{*}\right)$ have degree 2 .

On $W(\mathfrak{h})$, one defines a differential $d_{W}$ by 
- $\forall \gamma \in \wedge^{1}\left(\mathfrak{h}^{*}\right), d_{W} \gamma=d_{\mathfrak{h}} \gamma+\Gamma$, where $\Gamma$ equals $\gamma$ considered as an element of $S^{1}\left(\mathfrak{h}^{*}\right)$ and $d_{\mathfrak{h}}$ is the differential of the standard complex for the computation of the cohomology of $\mathfrak{h}$ with values in $\mathbb{R}$.

- $\forall \Omega \in S^{1}\left(\mathfrak{h}^{*}\right), d_{W} \Omega \in \wedge^{1}\left(\mathfrak{h}^{*}\right) \otimes S^{1}\left(\mathfrak{h}^{*}\right)$ and $d_{W} \Omega$ is the map from $\wedge^{1}(\mathfrak{h})$ to $S^{1}\left(\mathfrak{h}^{*}\right)$ defined by

$$
\forall X \in \mathfrak{h}, \quad\left(d_{W} \Omega\right)(X)=X \cdot \Omega,
$$

where $\cdot$ denotes the coadjoint action.

6.2 The Cartan Model. We assume now that $H$ is a compact connected (finite dimensional) Lie group acting on $M$ and that $\mathfrak{h}$ is its Lie algebra. We define the following differential algebra $(A, \partial)$ with $A=\left(\Omega(M) \otimes S\left(\mathfrak{h}^{*}\right)\right)^{H}$ and

- For all $\eta$ in $\Omega^{p}(M), \partial \eta=d \eta-\partial^{\prime} \eta$ were $d$ denotes the de Rham differential and $\partial^{\prime} \eta$ is the element of $\Omega^{p-1}(M) \otimes S^{1}\left(\mathfrak{h}^{*}\right)$ determined by

$$
\forall X \in \mathfrak{h} \quad\left(\partial^{\prime} \eta\right)(X)=i_{\tilde{X}} \eta
$$

where $i_{\tilde{X}}$ denotes the interior derivative with respect to $\tilde{X}$.

- For all $\phi$ in $S^{1}\left(\mathfrak{h}^{*}\right), \partial \phi=0$.

Let us recall that $(A, \partial)$ provides a model for the computation of the equivariant cohomology of $M$ with respect to $H$.

6.3 The BRST Model. The BRST model as described in [O-S-vB] and [Ka] is as follows. The algebra equals $W(h) \otimes \Omega(M)$, the differential equals

$$
d_{W} \otimes 1+1 \otimes d+\mathcal{L}-\partial^{\prime}
$$

where $\partial^{\prime}$ is defined above and $\mathcal{L}: \Omega^{p}(M) \rightarrow \Lambda^{1}(\mathfrak{h}) \otimes \Omega^{p}(M)$ is defined by $(\mathcal{L} \eta)(X)=\mathcal{L}_{\tilde{X}} \eta$, the Lie derivative of $\eta$ with respect to $\tilde{X}$. In [Ka] it is shown that this BRST differential can be obtained from the standard one, $d_{W} \otimes 1+1 \otimes d$, by an automorphism on the unrestricted BRST algebra $W(\mathfrak{h}) \otimes \Omega(M)$. Under this automorphism the basic subalgebra is mapped to the restricted BRST algebra $\left(S\left(\mathfrak{h}^{*}\right) \otimes \Omega(M)\right)^{H}$ and one obtains the Cartan model. In this subsection we will show how to obtain (8) as the BRST differential of section four by choosing the right symmetry group.

Assume that $M=B / C$, where $B$ and $C$ are Lie groups (not necessarily finite dimensional). This is always possible by putting $B=\operatorname{Diff} M$ and $C=\operatorname{Diff}_{m} M$ (where $\operatorname{Diff}_{m} M$ is the group of diffeomorphisms leaving the point $m$ invariant). Then $B$ acts on $M$ by left multiplication. Let $\mathfrak{b}$ be the Lie algebra of $B$. As before, we denote by $\sim: \mathfrak{b} \rightarrow \Gamma(M, T M)$ the Lie algebra morphism this action induces. During all this section, we also assume that the map

$$
\begin{aligned}
& A \otimes \mathfrak{b} \rightarrow \Gamma(M, T M) \\
& a \otimes X \mapsto a \tilde{X}
\end{aligned}
$$

is onto. Let $H$ be a finite dimensional connected compact Lie group. We assume moreover that we have a $C^{\infty}$ Lie groups morphisms $U$ from $H$ to $B$. Let $\mathfrak{h}$ be the 
Lie algebra of $H$ and $u: \mathfrak{h} \rightarrow \mathfrak{b}$ the differential of $U$ at the unity. So $H$ acts on

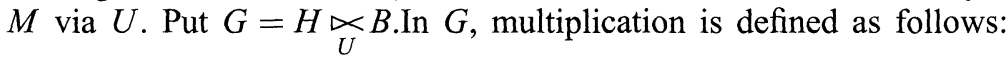

$$
\forall\left(h h^{\prime}\right) \in H^{2}, \forall\left(b, b^{\prime}\right) \in B^{2}, \quad(h, b)\left(h^{\prime}, b^{\prime}\right)=\left(h h^{\prime}, U\left(h^{\prime-1}\right) b U\left(h^{\prime}\right) b^{\prime}\right) .
$$

This multiplication is such that the following map defines a group action of $G$ on $M$ :

$$
G \times M \rightarrow M, \quad(h, b) \cdot m \mapsto h \cdot(b \cdot m) .
$$

Let $\mathfrak{g}$ be the Lie algebra of $G$. We have $\mathfrak{g}=\mathfrak{h} \underset{u}{\ltimes} \mathfrak{b}$. In $\mathfrak{g}$, the Lie bracket is given by

$$
\begin{aligned}
\forall\left(H, H^{\prime}\right), \forall\left(D, D^{\prime}\right) \in B,\left[H+D, H^{\prime}+D^{\prime}\right]= & {\left[H, H^{\prime}\right]+\left[D, u\left(H^{\prime}\right)\right] } \\
& +[u(H), D]+\left[D, D^{\prime}\right] .
\end{aligned}
$$

Put $F=A \otimes \mathfrak{g}$ and $E=A \otimes \mathfrak{b}$. As the action of $G$ and $B$ are transitive, we have the following exact sequences:

$$
\begin{gathered}
\{0\} \rightarrow P \rightarrow F \stackrel{\Phi}{\rightarrow} \Gamma(M, T M) \rightarrow\{0\} \\
\{0\} \rightarrow Q \rightarrow E \stackrel{\Psi}{\rightarrow} \Gamma(M, T M) \rightarrow\{0\},
\end{gathered}
$$

where $\Phi$ and $\Psi$ are given by

$$
\begin{array}{ll}
\forall f \in A, \forall H \in \mathfrak{h}, \forall D \in \mathfrak{b} \quad & \Phi(f(H+D))=f(\widetilde{u(H)}+\tilde{D}) \\
& \Psi(f D)=f \tilde{D} .
\end{array}
$$

We have $F=(A \otimes \mathfrak{h}) \oplus E$ and

$$
\begin{aligned}
P & =\{f(H+D) \in A \otimes F \mid f(\widetilde{u(H)}+\tilde{D})=0\} \\
& =\{f(-H+u(H)) \mid H \in \mathfrak{h}\} \oplus\{f D \mid f \tilde{D}=0\} \\
& \simeq(A \otimes \mathfrak{h}) \oplus Q .
\end{aligned}
$$

Then,

$$
\bigoplus_{q}\left(S_{A}^{q}(P)\right)^{*}=\bigoplus_{r}\left(S_{A}^{r}(Q)\right)^{*} \bigotimes_{A}\left(S_{A}\left((A \otimes \mathfrak{h})^{*}\right) \simeq \bigoplus_{r}\left(S_{A}^{r}(Q)\right)^{*} \bigotimes_{\mathbb{R}} S\left(\mathfrak{h}^{*}\right)\right.
$$

From this, we obtain

$$
\bigoplus_{p, q} \operatorname{Hom}_{A}\left(\Lambda_{A}^{p} F,\left(S_{A}^{q}(P)^{*}\right) \simeq \bigoplus_{p, q} \operatorname{Hom}_{A}\left(\Lambda_{A}^{p} E, W(\mathfrak{h}) \otimes\left(S_{A}^{q}(Q)^{*}\right),\right.\right.
$$

where $W(\mathfrak{h})$ is the Weil algebra of $\mathfrak{h}$.

Recall that $(\mathcal{C}(G), D)$ (respectively $(\mathcal{C}(B), \delta)$ ) denotes the BRST complex associated to the action of $G$ (respectively $B$ ). We are going to give the explicit expression for $D$. For this, we identify $\mathcal{C}(G)$ with $\oplus_{p, q} \operatorname{Hom}_{A}\left(\Lambda_{A}^{p} E, W(\mathfrak{h}) \otimes\left(S_{A}^{q}(Q)^{*}\right)\right.$. Then, the expression below come from straightforward computations if one remembers the following asymmetry in our identification: an element $H$ in $S^{1}(\mathfrak{h})$ represents the pair $(H,-u(H))$ but an element $H$ of $\Lambda^{1}(\mathfrak{h})$ represents the pair $(H, 0)$. Let $\omega$ be in $\operatorname{Hom}_{A}\left(\Lambda_{A}^{p} E,\left(\Lambda^{r}\left(\mathfrak{h}^{*}\right) \otimes\left(S^{n}\left(\mathfrak{h}^{*}\right)\right) \otimes\left(S_{A}^{q} Q\right)^{*}\right)\right.$. Clearly, $D \omega$ decomposes into five 
parts $D \omega=\omega_{(1)}+\omega_{(2)}+\omega_{(3)}+\omega_{(4)}+\omega_{(5)}$. While reading the following definitions one should keep in mind the following anology with (8); $\omega_{(1)}+\omega_{(4)}$ gives $1 \otimes d$ as in section five, $\omega_{(5)}$ equals $d_{W} \otimes 1, \omega_{(2)}$ leads to $\mathcal{L}$ and $\omega_{(3)}$ gives $-\partial^{\prime}$ (see also the remark at the end of this section).

- $\omega_{(1)} \in \operatorname{Hom}_{A}\left(\Lambda_{A}^{p+1} E,\left(\Lambda^{r}\left(\mathfrak{h}^{*}\right) \otimes S^{n}\left(\mathfrak{h}^{*}\right)\right) \otimes\left(S_{A}^{q} Q\right)^{*}\right)$ and $\forall\left(\mathcal{X}_{1}, \ldots, \mathcal{X}_{p+1}\right) \in E, \forall\left(H_{1}, \ldots, H_{r}\right) \in \mathfrak{h}$,

$$
\begin{aligned}
\omega_{(1)} & \left(\mathcal{X}_{1}, \ldots, \mathcal{X}_{p+1}\right)\left(H_{1}, \ldots, H_{r}\right) \\
= & \sum_{i} \mathcal{X}_{i} \cdot\left[\omega\left(\mathcal{X}_{1}, \ldots, \widehat{\mathcal{X}}_{i}, \ldots, \mathcal{X}_{p+1}\right)\left(H_{1}, \ldots, H_{r}\right)\right](-1)^{i-1} \\
& +\sum_{i<j} \omega\left(\left[\mathcal{X}_{i}, \mathcal{X}_{j}\right], \mathcal{X}_{1} \ldots, \widehat{\mathcal{X}}_{i}, \ldots, \widehat{\mathcal{X}}_{j}, \ldots, \mathcal{X}_{p+1}\right)\left(H_{1}, \ldots, H_{r}\right)(-1)^{i+j}
\end{aligned}
$$

- $\omega_{(2)} \in \operatorname{Hom}_{A}\left(\Lambda_{A}^{p} E,\left(\Lambda^{r+1}\left(\mathfrak{h}^{*}\right) \otimes S^{n}\left(\mathfrak{h}^{*}\right)\right) \otimes\left(S_{A}^{q} Q\right)^{*}\right)$ and $\forall\left(\mathcal{X}_{1}, \ldots, \mathcal{X}_{p}\right) \in E, \forall\left(H_{1}, \ldots, H_{r+1}\right) \in \mathfrak{h}$,

$$
\begin{aligned}
\omega_{(2)}\left(\mathcal{X}_{1}, \ldots, \mathcal{X}_{p}\right)\left(H_{1}, \ldots, H_{r+1}\right) \\
=u\left(H_{i}\right) \cdot\left[\omega\left(\mathcal{X}_{1}, \ldots, \mathcal{X}_{p}\right)\left(H_{1}, \ldots, \widehat{H}_{i}, \ldots, H_{r+1}\right)\right](-1)^{p+i-1} \\
\quad+\sum_{j=1}^{p} \sum_{i=1}^{r+1} \omega\left(\left[\mathcal{X}_{j}, H_{i}\right], \mathcal{X}_{1}, \ldots, \widehat{\mathcal{X}}_{j}, \ldots, \mathcal{X}_{p}\right) \\
\quad \times\left(H_{1}, \ldots, \widehat{H}_{l}, \ldots, H_{r+1}\right)(-1)^{p+i+j} .
\end{aligned}
$$

Note that $u\left(H_{i}\right)$ acts trivially on $S\left(\mathfrak{h}^{*}\right)$.

- $\omega_{(3)} \in \operatorname{Hom}_{A}\left(\Lambda_{A}^{p-1} E,\left(\Lambda^{r}\left(\mathfrak{h}^{*}\right) \otimes S^{n+1}\left(h^{*}\right)\right) \otimes\left(S_{A}^{q} Q\right)^{*}\right.$ and $\forall\left(\mathcal{X}_{1}, \ldots, \mathcal{X}_{p}\right) \in E, \forall\left(H_{1}, \ldots, H_{n+1}\right) \in \mathfrak{h}$,

$\omega_{(3)}\left(\mathcal{X}_{1}, \ldots, \mathcal{X}_{p}\right)\left(H_{1}, \ldots, H_{n+1}\right)$

$=-\sum_{i=1}^{n+1} \omega\left(u\left(H_{i}\right), \mathcal{X}_{1}, \ldots, \mathcal{X}_{p-1}\right)\left(H_{1}, \ldots, \widehat{H}_{i}, \ldots, H_{n+1}\right)$

- $\omega_{(4)} \in \operatorname{Hom}_{A}\left(\Lambda_{A}^{p-1} E,\left(\Lambda^{r}\left(\mathfrak{h}^{*}\right) \otimes S^{n}\left(\mathfrak{h}^{*}\right)\right) \otimes\left(S_{A}^{q+1} Q\right)^{*}\right)$ and $\left(\mathcal{X}_{1}, \ldots, \mathcal{X}_{p-1}\right) \in E, \forall\left(\mathcal{Q}_{1}, \ldots, \mathcal{Q}_{q+1}\right) \in Q$

$\omega_{(4)}\left(\mathcal{X}_{1}, \ldots, \mathcal{X}_{p-1}\right)\left(\mathcal{Q}_{1}, \ldots, \mathcal{Q}_{q+1}\right)$

$$
\left.=-\sum_{i=1}^{q+1} \omega\left(\mathcal{Q}_{i}\right), \mathcal{X}_{1}, \ldots, \mathcal{X}_{p-1}\right)\left(\mathcal{Q}_{1}, \ldots, \widehat{\mathcal{Q}}_{i}, \ldots, \mathcal{Q}_{q+1}\right) .
$$

- $\omega_{(5)} \in \operatorname{Hom}_{A}\left(\Lambda_{A}^{p} E, W^{r+n+1}(\mathfrak{h}) \otimes\left(S_{A}^{q} Q\right)^{*}\right)$ and $\forall\left(\mathcal{X}_{1}, \ldots, \mathcal{X}_{p}\right) \in E, \quad \omega_{(5)}\left(\mathcal{X}_{1}, \ldots, \mathcal{X}_{p}\right)=\left(d_{W} \otimes \mathrm{id}\right)\left(\omega\left(\mathcal{X}_{1}, \ldots, \mathcal{X}_{p}\right)\right)$. 
As in [Ka] and [O-S-vB], we restrict the BRST algebra to obtain a model for equivariant cohomology. We define

$$
\mathcal{C}(G)_{\mathrm{rest}}=\left[\bigoplus_{p, q} \operatorname{Hom}_{A}\left(\Lambda_{A}^{p} E, S\left(\mathfrak{h}^{*}\right) \otimes\left(S_{A}^{q} Q\right)^{*}\right)\right]^{H} .
$$

One can see easily that $\mathcal{C}(G)_{\text {rest }}$ is invariant under $D$. Moreover, on $\mathcal{C}(G)_{\text {rest }}$, the expression of $D$ is slightly simpler. Let $\omega$ be in $\left[\operatorname{Hom}_{A}\left(A_{A}^{p} E, S^{n}\left(\mathfrak{h}^{*}\right) \otimes\left(S_{A}^{q} Q\right)^{*}\right)\right]^{H}$. With the previousnotations, we have

$$
D \omega=\omega_{(1)}+\omega_{(3)}+\omega_{(4)} .
$$

On $\mathcal{C}(G)_{\text {rest }}$, we are going to put a double complex structure slightly different than the one we had before. The first degree of $\omega$ is $p+q+2 n$ and its second degree is $q$. We put

$$
D^{\prime}(\omega)=\omega_{(1)}+\omega_{(3)} \text { and } D^{\prime \prime}(\omega)=\omega_{(4)} .
$$

$D^{\prime}$ (respectively $D^{\prime \prime}$ ) increases the first (respectively the second) degree by one and leaves the second (respectively the first) degree invariant.

Lemma 6.3.1. $D^{\prime 2}=D^{\prime \prime 2}=0$ and $D^{\prime} D^{\prime \prime}=-D^{\prime \prime} D^{\prime}$.

The proof of Lemma 6.3.1 consists in computations similar to those appearing in Proposition 4.0.1. We don't reproduce them here.

So we have defined a double complex structure on $\mathcal{C}(G)_{\text {rest }}$. We are now going to prove the theorem linking BRST cohomology and equivariant cohomology.

Theorem 6.3.2. Assume that there exists a A-module $R$ which is also $H$-invariant such that $Q \oplus R=E$. Then, the map $\mathcal{F}$ from $\left[\left(\Omega(M) \otimes S\left(\mathfrak{h}^{*}\right)\right)^{H}, \partial\right]$ to $\left[\left(\bigoplus_{q} \operatorname{Hom}_{A}\left(\Lambda_{A}^{p} E, S\left(\mathfrak{h}^{*}\right) \otimes\left(S_{A}^{q} Q\right)^{*}\right)\right)^{H}, D\right]$ defined by

$$
\begin{array}{r}
\forall \sigma \in\left(\Omega^{p}(M) \otimes S\left(\mathfrak{h}^{*}\right)\right)^{H}, \forall\left(\mathcal{X}_{1}, \ldots, \mathcal{X}_{p}\right) \in E, \\
\mathcal{F}(\sigma)\left(\mathcal{X}_{1}, \ldots, \mathcal{X}_{p}\right)=\sigma\left(\Psi\left(\mathcal{X}_{1}\right), \ldots, \Psi\left(\mathcal{X}_{p}\right)\right)
\end{array}
$$

is a quasi-isomorphism.

Remarks. The hypothesis of the theorem is satisfied in the following two cases:

- If $B$ is finite dimensional. Then the kernel $V$ of the vector bundle map $\phi: W=$ $M \times \mathfrak{b} \rightarrow T(M)$ is a vector bundle. If we put an $H$-invariant inner product $\langle$, on the fibres $\mathfrak{g}$ of $W$, the orthogonal complement of $V$ with respect to $\langle\rangle,, V^{\perp}$, is a vector bundle such that $V \oplus V^{\perp}=W$. Then, we can take $R=\Gamma\left(M, V^{\perp}\right)$.

- If $B=\operatorname{Diff}(M)$, then the decomposition given at the end of 3.1 is $H$-invariant.

Proof of Theorem 6.3.2. A small computation shows that $\mathcal{F}$ is a morphism of complexes. Moreover, $\mathcal{F}$ is injective and its image is

$$
\operatorname{Im} \mathcal{F}=\bigoplus_{p}\left(\bigwedge_{A}^{p}(E / Q)^{*} \otimes S\left(\mathfrak{h}^{*}\right)\right)^{H}
$$


For convenience, we put $\mathcal{I}=(\operatorname{Im} \mathcal{F}, D)$. Then $\mathcal{I}$ is isomorphic to $[(\Omega(M) \otimes$ $\left.\left.S\left(\mathfrak{h}^{*}\right)\right)^{H}, \partial\right]$. Let us prove that $H^{\bullet}(\mathcal{I})=H^{\bullet}\left(\mathcal{C}(G)_{\text {rest }}, D\right)$. We proceed as for Theorem 5.0.1: we use the double complex structure of $\mathcal{C}(G)_{\text {rest }}$. We first compute the cohomology of $\mathcal{C}(G)_{\text {rest }}$ with respect of $D^{\prime \prime}$. We clearly have $H^{0}\left(\mathcal{C}(G)_{\text {rest }}, D^{\prime \prime}\right)=$ $\bigoplus_{p}\left(\bigwedge_{A}^{p}(E / Q)^{*} \otimes S\left(\mathfrak{h}^{*}\right)\right)^{H}$. Let us now prove that $H^{q}\left(\mathcal{C}(G)_{\text {rest }}, D^{\prime \prime}\right)=\{0\}$ if $q>0$. For this, we use the $H$-invariant decomposition $E=Q \oplus R$. Let $q>0$. Then Ker $D^{\prime \prime} \cap\left(\bigoplus_{p} \operatorname{Hom}_{A}\left(\Lambda_{A}^{p} E, S\left(\mathfrak{h}^{*}\right) \otimes\left(S_{A}^{q} Q\right)^{*}\right)\right)^{H}$. is isomorphic to $\left(\bigoplus_{p} \operatorname{Hom}_{A}\left(\Lambda_{A}^{p}(E / Q)\right.\right.$, $\left.\left.S\left(\mathfrak{h}^{*}\right) \otimes\left(S_{A}^{q} Q\right)^{*}\right)\right)^{H}$. Let us prove that any element of $\left(\bigoplus_{p} \operatorname{Hom}_{A}\left(\Lambda_{A}^{p}(E / Q), S\left(\mathfrak{h}^{*}\right) \otimes\right.\right.$ $\left.\left.\left(S_{A}^{q} Q\right)^{*}\right)\right)^{H}$ is a coboundary. Let $\omega$ be such an element. We construct an element $\Omega$ in $\left(\bigoplus_{p} \operatorname{Hom}_{A}\left(\Lambda_{A}^{p+1} E, S\left(\mathfrak{h}^{*}\right) \otimes\left(S_{A}^{q-1} Q\right)^{*}\right)\right)$ such that $D^{\prime \prime} \Omega=\omega$ exactly as we did in the proof of Theorem 5.0.1. But as the decomposition we use is $H$-invariant, it is easy to see that our $\Omega$ is also $H$-invariant. So we have proved $H^{q}\left(\mathcal{C}(G)_{\text {rest }}, D^{\prime \prime}\right)=\{0\}$. From this we deduce the following isomorphisms:

$$
\begin{aligned}
H^{\bullet}\left(\mathcal{C}(G)_{\text {rest }}, D^{\prime \prime}\right) & \simeq H^{\bullet}\left[\bigoplus_{p}\left(\wedge^{p}(E / Q)^{*} \otimes S\left(\mathfrak{h}^{*}\right)\right)^{H}, D^{\prime}\right] \\
& \simeq H^{\bullet}\left[\left(\Omega(M) \otimes S\left(\mathfrak{h}^{*}\right)\right)^{H}, \partial\right] .
\end{aligned}
$$

This finishes the proof of Theorem 6.3.2.

As we know that the (restricted) BRST complex for topological theories equals the Cartan model for equivariant cohomology, we now come to the corollary we are aiming at:

Corollary 6.3.3. Let $H$ be a compact Lie group acting on a manifold $M$. Let $\mathfrak{h}$ be the Lie algebra of $H$. The restricted BRST complex $\mathcal{C}(H \bowtie \operatorname{Diff} M)_{\text {rest }}$ is quasiisomorphic to the Cartan model $\left[\left(\Omega(M) \otimes S\left(\mathfrak{h}^{*}\right)\right)^{H}, \partial\right]$.

The corollary is an immediate consequence of Theorem 6.3 .2 if we write $M=$ $\operatorname{Diff}(M) / \operatorname{Diff}_{m}(M)$.

Remark. By the same techniques, one can show that the complex $\mathcal{C}(H \bowtie$ $\operatorname{Diff}(M))$ is isomorphic to the unrestricted BRST complex for topological theories with algebra $\Omega(M) \otimes W(\mathfrak{h})$ and differential (8).

\section{Conclusions}

We have established a link between two different definitions of the BRST complex. The first one is the BRST complex for constrained Hamiltonian systems ([F-H$\mathrm{S}-\mathrm{T}],[\mathrm{K}-\mathrm{S}],[\mathrm{S}])$. The second one concerns the BRST construction associated to cohomological field theories ([O-S-vB], $[\mathrm{Ka}])$. Actually, we linked the ghost parts (which are the most important parts) of both complexes.

Extending the first BRST theory to constraints coming from infinite dimensional groups and applying this to a semi-direct product of the type $H \bowtie \operatorname{Diff}(M)$, we get the unrestricted BRST algebra for cohomological field theories. Restricting the former BRST algebra gives the (restricted) complex for cohomological field theories leading to equivariant cohomology. 
Acknowledgments. We would like to thank Y. Benoist, M. Duflo, H. Duistermaat, O. Mathieu and G. Tuynman for helpful discussions. The first author expresses here gratitude towards H. Duistermaat, Y. Karshon and S. Sternberg for introducing her to symplectic geometry.

\section{References}

[B-V] Batalin, I.A., Vilkovisky, G. A.: Relativistic S-matrix of dynamical systems with boson and fermion constraints. Phys. Lett. 69B, 309 (1977)

[C] Cartan H.: Notions d'algèbres différentielles: Applications aux groupes de Lie et aux vari ét és où opèrent un groupe de Lie. Colloque de Topologie, Bruxelles (1950), pp. $15-27$

[F] Fel'dman, G.L.: Global dimension of rings of differential differential opeators. Trans. Moscow. Math. Soc. No 1, 123-147 (1982)

[F-F] Fradkin, E.S., Fradkina, T.E.: Quantization of relativisitc systems with boson and fermion first and second class constraints. Phys. Lett. 72B, 343 (1987)

[F-H-S-T] Fisch, J. Henneaux, M., Stasheff, J. Teitelboim C.: Existence, uniqueness and cohomology of the classical BRST charge with Ghosts of Ghosts. Commun. Math. Phys. 120, 379-407 (1989)

[H] Henneaux, M.: Hamiltonian form of the path integral for theories with a gauge freedom. Phys. Rep. 126, 1 (1985)

[H-T] Henneaux, -M., Teitelboim, C.: BRST cohomology in classical mechanics. Commun. Math. Phys. 115, 213 (1988)

[H-S] Hilton, P.J., Stammbach, U.: A course in homological algebra. Graduate Texts in Mathematics. Berlin, Heidelberg, NewYork: Springer, (1971)

[Hu] Husemoller: Fiber bundles. Graduate Texts in Mathematics, 1966

[Ka] J. Kalkman: BRST model for equivariant cohomology and representatives for equivariant Thom classes. Commun. Math. Phys. 153, 447-463 (1993)

[K-S] Kostant, B., Sternberg, S.: Symplectic reduction, BRS cohomology, and infinite dimensional Clifford algebras. Ann. Phys. 176, No 1, 49-113 (1987)

[M] Malliavin, M.P.: Algèbre homologique et opérateurs différentiels, Ring theory. Lect. Notes in Math. 1328, Berlin, Heidelberg, NewYork: Springer 1988, pp 172-186

[O-S-vB] Ouvry, S., Stora, R., van Baal, P.: Algebraic characterization of topological Yang Mills. Phys. Lett. 220B, 1590 (1989)

[S] Stasheff, J.: Homological reduction of constrained Poisson algebras. UNC-MATH Preprint, February, 1989

[We] Wells, R.O.: Differential analysis on complex manifolds. NewYork: Prentice-Hall 1973

[Wi] Witten, E.: Introduction to cohomological field theories. Int. J. Mod. Phys. A6, 2775 (1991) 